\title{
Correction to: Aberrant Expression of Sodium-Potassium-Chloride Cotransporter in Endometriosis
}

Inha Lee ${ }^{1,2} \cdot$ Myung Jae Jeon ${ }^{3} \cdot$ Jeong Sook Kim ${ }^{4} \cdot$ Ji Hyun Park ${ }^{2,5} \cdot$ Bo Hee Won ${ }^{2,5} \cdot$ Heeyon Kim ${ }^{1,2} \cdot$ Jae Hoon Lee ${ }^{2,5}$. Bo Hyon Yun ${ }^{1,2}$. Joo Hyun Park ${ }^{2,5}$. Seok Kyo Seo ${ }^{1,2}$. Young Sik Choi ${ }^{1,2} \cdot$ SiHyun Cho ${ }^{2,5} \cdot$ Byung Seok Lee ${ }^{1,2}$

Published online: 24 March 2021

(C) Society for Reproductive Investigation 2021

\section{Correction to: Reproductive Sciences} https://doi.org/10.1007/s43032-021-00531-4

The article was updated to include an Article Note that Inha Lee and Dr. Myung Jae Jeon equally contributed to this work.

Publisher's Note Springer Nature remains neutral with regard to jurisdictional claims in published maps and institutional affiliations.

The online version of the original article can be found at https://doi.org/ 10.1007/s43032-021-00531-4

$\triangle$ SiHyun Cho

sihyuncho@yuhs.ac

1 Department of Obstetrics and Gynecology, Severance Hospital, Yonsei University College of Medicine, Seoul, South Korea

2 Institute of Women's Life Medical Science, Yonsei University College of Medicine, Seoul, South Korea

3 Department of Obstetrics and Gynecology, Seoul National University Hospital, Seoul National University College of Medicine, Seoul, South Korea

4 Department of Obstetrics and Gynecology, University of Ulsan College of Medicine, Ulsan University Hospital, Ulsan, South Korea

5 Department of Obstetrics and Gynecology, Gangnam Severance Hospital, Yonsei University College of Medicine, 211 Eonju-ro, Gangnam-gu, Seoul 06273, South Korea 